\title{
Responsable de sa vie, responsable devant sa mort?
}

\author{
Christian Lalive d'Epinay \\ Dr sc. écon. et soc., professeur honoraire de I'Université de Genève
}

\author{
Les contours d'une éthique qui ne prétend pas imposer tel ou tel choix en fin de \\ vie mais qui pose un principe de responsabilité devant la mort et revendique le \\ droit au choix.
}

Mesurons le chemin parcouru: au début des années 1990, dans le cadre de la $3^{\text {e }}$ Commission fédérale sur les personnes âgées, il fallut batailler ferme pour qu'on y traitât non seulement de l'accompagnement en fin de vie, mais aussi des suicides de vieillards, de l'euthanasie et des positions défendues par Exit. Malgré des menaces de démission, la large majorité des membres tint bon et le rapport, publié en 1995 dans les trois langues fédérales, comprend un chapitre 13 intitulé "L'euthanasie, état du dossier»; sa conclusion, en forme de recommandation, appelle à un large débat public «dans la recherche d'une éthique collective partagée» [1].

\section{Le suicide assisté: un débat publique actuel}

Vingt années se sont écoulées: le vœu de voir le débat porté sur la place publique est largement exaucé aujourd'hui; il ne se passe guère de semaine sans que quotidiens et hebdomadaires, ainsi que la presse spécialisée ne commentent tel cas, telle prise de position, telle décision. On distingue aujourd'hui plus clairement euthanasie passive et active, et on parle sans détour de suicide assisté. Car c'est bien de cela qu'il s'agit, du droit de se donner la mort et de se voir proposer les moyens de le faire «dans la dignité».

Où en est-on aujourd'hui en Suisse en ce qui concerne «la recherche d'une éthique collective partagée»? Depuis longtemps déjà le suicide n'y est plus un délit, quand au suicide assisté, il est toléré (selon l'art. 115 du Code pénal est condamnable celui qui incite au suicide "pour un motif égoïste», mais l'article ne se prononce pas en ce qui concerne un accompagnement altruiste; qui ne dit mot consent!); en revanche l'euthanasie active directe (tuer quelqu'un à sa demande) reste, elle, condamnable (l'art. 114 prévoit une peine de prison pouvant aller jusqu'à cinq ans). Dans les faits, sous l'impulsion principale d'Exit, le suicide assisté a aujourd'hui droit de cité, mais comme l'acquisition d'une substance létale nécessite une ordonnance, le candidat, de même que ceux qui l'assistent, doivent se soumettre aux Directives de l'ASSM, (inscrites dans le Code déontologique de la FMH): la présence d'une maladie létale active sera attestée médicalement, la volonté de se donner la mort devra être exprimée et confirmée par une personne en possession de ses facultés cognitives, qui ne souffre pas de dépression ni ne subit quelque influence indue. Par ailleurs, au moment de passer à l'acte, celle-ci doit le commettre elle-même, sous peine pour celui qui l'assisterait de tomber sous le coup de l'art. 114 du Code pénal.

L'exigence de garde-fous est en soi légitime; observons cependant que ces prérequis imposent à la personne qui décide de mettre un terme à sa vie un véritable parcours du combattant [2]. "Pourquoi faut-il devoir ainsi «mériter» le droit de mourir?» m’a confié un vieillard [3].

\section{Le système d'aujourd'hui a des brèches}

Des brèches s'ouvrent pourtant dans le système actuel. Il arrive que les délais imposés, qui ne suspendent pas le cours de la maladie, fassent que le jour venu, la personne ne soit plus à même d'avaler la potion ni de déclencher l'injection. Ce médecin qui en pareille situation s'autorisa de faciliter le geste du vieillard a été acquitté (déc. 2010); très récemment (avril 2014), suite à un recours après une première condamnation, un tribunal de Neuchâtel acquitta un autre médecin accusé lui d'avoir prescrit à un patient une substance létale sans avoir suffisamment vérifié la gravité de son état de santé [4].

Le 17 juin 2012, le Canton de Vaud a connu un vote historique par lequel les citoyens ont autorisé l'entrée d'Exit dans les EMS, quand bien même les conditions imposées soient plus rigides que ce que souhaitait cette association. Et voilà aussi que depuis le printemps 2014, cette «Association pour le droit de mou- 


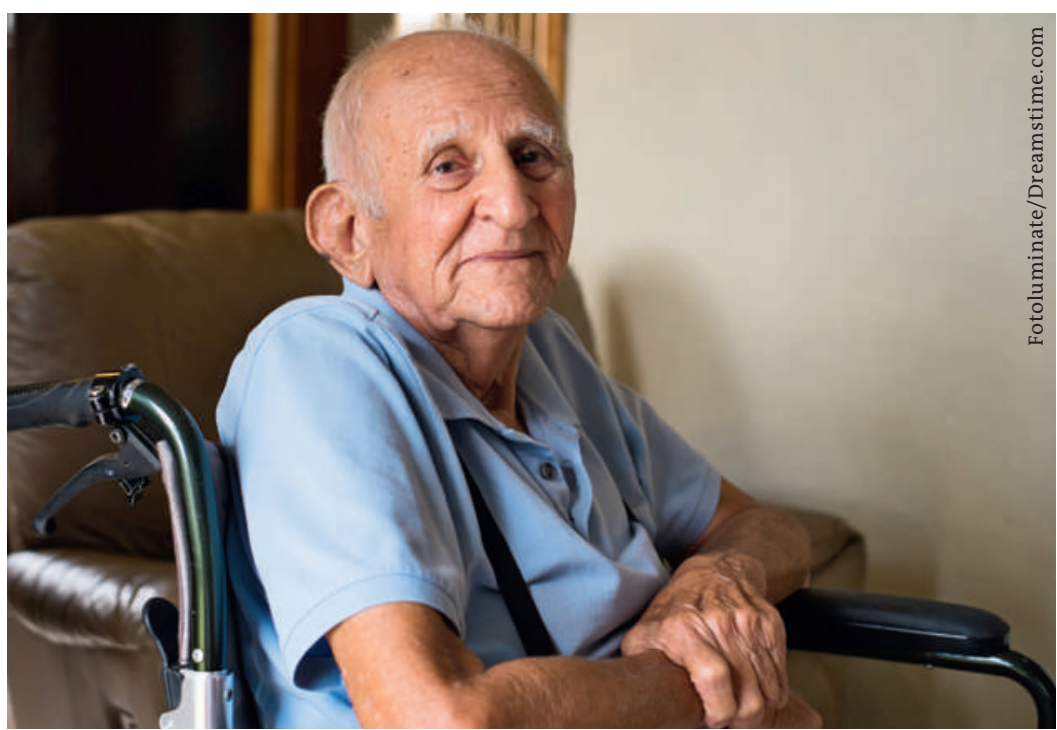

Exit revendique le droit d'accompagner des vieillards souffrant de «polypathologie du grand âge»: une rupture inquiétante?

rir dans la dignité» met en question le critère de maladie létale et revendique le droit d'accompagner des vieillards souffrant de "polypathologie du grand âge». De facto, le champ d'action d'Exit s'élargit ainsi potentiellement à tous les grands vieillards puisque, comme le rappelle B. Kiefer, à un stade avancé de la vieillesse, presque tous souffrent de polypathologie. Le rédacteur en chef de la Revue Médicale Suisse y voit «une rupture inquiétante» [5].

Commençons par relever quelques faits. En Suisse, toujours au cours des vingt dernières années, l'espérance de vie des hommes a augmenté de six ans et celle des femmes de presque quatre ans, pour atteindre aujourd'hui respectivement 80 et 85 ans. Observons cette longévité croissante d'une autre manière: celle de l'âge au décès. Du total des défunts de l'année 1992, 18\% avaient plus de 85 ans; en 2010, cette proportion était de $41 \%$ ! Ces toutes prochaines années - si ce n'est déjà le cas - un décédé sur deux aura connu le très grand âge [6].

\section{La vieillesse au double visage d'un Janus}

Pour les personnes qui cheminent dans la vieillesse (parmi lesquelles je me compte), la réalité qu'évoquent ces données présente le double visage de Janus. Face, le dieu des transitions et des renaissances nous sourit, d'autant que le gain de vie est composé de plus de mois en bonne santé (relative) que de mois porteurs de maladies et de handicaps. Pile, le sourire devient rictus, car plus on avance dans le grand âge, plus le risque grandit de devoir terminer sa vie en souffrant de l'une ou l'autre forme de dépendance chronique, physique et surtout psychique [7].
Tel semble aujourd'hui être le prix à payer pour les progrès de la médecine. Dans certains domaines, indéniablement, celle-ci a rajouté «de la vie aux années» - celles et ceux qui ont récupéré leur mobilité grâce à une prothèse de la hanche, ou la vue suite à une opération de la cataracte, se réjouissent d'une qualité de vie retrouvée! Mais à force de parvenir à contenir, à juguler, parfois même à éradiquer tout un ensemble de maladies grâce tant à la prévention qu'au traitement, nous voilà au grand âge livrés à la voracité des hydres que sont les maladies dégénératives, maladies de Parkinson et d'Alzheimer et autre polyarthrite, qui ne tuent qu'à petit feu, mais qui distillent dans les corps la souffrance au quotidien et transforment l'être humain au point de le rendre étranger à lui-même, biologiquement vivant mais zombie. Devant ces hydres, à ce jour, la science reste balbutiante, impuissante ou presque.

C'est, je crois, dans ce contexte qu'il faut comprendre les résultats d'un sondage réalisé au mois d'août 2014, peu après la décision d'Exit d'élargir son champ d'action, à la demande du journal protestant Evangelisch-Reformierte-Zeitung [8]. Ce sondage a porté sur 1004 (soyons précis!) personnes âgées de 15 à 75 ans en Suisse alémanique et romande. Tout d'abord, rappelons les limites de ce type d'exercice. Il s'agit d'avis recueillis par téléphone, ce qui suppose un taux très élevé de refus de répondre, et aussi que certains groupes de la population sont sous-représentés, parce que plus difficiles à atteindre. C'est très vraisemblablement le cas de la population d'immigration récente. Prenons acte de l'absence de la Suisse italienne; on peut cependant s'étonner de l'exclusion des personnes âgées (de plus de 75 ans), qui sont les plus directement concernées par la thématique! Peut-être est-ce là la raison? On sait aussi que l'entretien téléphonique avec les aînés est souvent malaisé (problèmes d'audition, etc.). Voilà qui permet de mieux cerner la population enquêtée, mais ajoutons qu'on ne sait rien de la qualité de la réflexion qui sous-tend les réponses données. Regrettons ici l'absence de questions qui auraient permis de vérifier, par ex., si la personne interrogée connaît ou non Exit et si elle est au courant du récent changement de ses statuts? Tout ce qu'on sait, c'est que les membres d'Exit présents dans l'échantillon sont très peu nombreux (2\%).

\section{Tendances frappantes face au suicide assisté}

Dès lors, dans la lecture des résultats, on ne s'arrêtera pas au détail du pourcentage, à la précision arithmétique bien illusoire! Nous intéresse ici la vue d'ensemble: y voit-on se dégager dans l'opinion publique 
des lignes de forces, des tendances massives? J'en relève quatre.

La première est l'approbation forte de la demande d'Exit de ce que les personnes âgées puissent bénéficier d'un accès facilité à des moyens de se donner la mort. C'est le fait de deux répondants sur trois, et ce résultat se retrouve dans tous les groupes, hommes et femmes, Alémaniques et Romands, jeunes et âgés, personnes à revenus élevés et personnes à revenus plus modestes, et - ce qui est peut-être le plus surprenant tant parmi les catholiques que parmi les réformés.

La deuxième est le rejet prononcé de l'enseignement traditionnel des Eglises en cette matière. Des majorités des deux-tiers aux trois-quarts réfutent les énoncés théologiques condamnant le suicide, à savoir: la vie étant un don de Dieu, donc l'homme n'a pas le droit de se l'ôter, ou, autre variante, l'homme ayant été créé à l'image de Dieu, se tuer serait une profanation. Et sept personnes sur dix estiment que «la religion ne doit pas énoncer des directives sur la question du suicide».

Troisième ligne de force: ce rejet de l'enseignement traditionnel est accompagné d'une conviction forte, partagée par trois personnes sur quatre, à savoir que l'être humain est un être responsable de soi, et que cette responsabilité s'étend jusqu'à la mort.

Quatrième ligne de force: ces trois tendances précédentes - libéralisation des moyens de suicide, rejet de l'enseignement religieux traditionnel, affirmation du principe de responsabilité individuelle - ne sont pas seulement le fait d'une large majorité, elles s'expriment aussi de manière très consensuelle dans tous les groupes socioculturels. En cette matière, pas de Röstigraben, pas d'opposition entre groupes économiques (tout au plus peut-on relever que ces tendances sont un peu plus marquées dans les agglomérations qu'ailleurs), ni de guerre des sexes. Pas plus que de guerre de religions: catholiques et réformés ne sont séparés ici et là que par quelques petits pour cents. (Les personnes sans attache confessionnelle présentant des majorités un peu plus fortes.)

Ainsi l'éthique chrétienne traditionnelle qui fait de la vie un absolu, car elle est donnée par un Dieu qui en est le maître de la conception à la tombe, cette éthique souffre aujourd'hui d'obsolescence. Cette vision de l'homme et de la vie (d'ailleurs partagée par les trois religions abrahamiques) a dominé l'Occident pendant près de deux millénaires, et aujourd'hui encore des autorités ecclésiastiques (particulièrement celle de l'Eglise catholique mais aussi celles du protestantisme dit "évangélique») condamnent toute atteinte au dogme et, là où elles en ont le pouvoir, veillent à ce que le dogme reste traduit dans les législations nationales. L'enquête de Reformiert met en lumière le déphasage radical entre le discours de ces hiérarchies et une très large majorité de l'opinion publique. Tout en montrant aussi qu'une minorité lui reste acquise. Or la qualité d'une démocratie réside dans le respect de ses minorités.

\section{Chaque choix a droit à un égal respect}

L'étude confirme l'affirmation d'une éthique qui est l'héritière des Lumières et qui a accompagné depuis trois siècles le processus de sécularisation, mais dont la propagation a vraisemblablement connu une forte accélération au cours des dernières décennies. Cette éthique connaît diverses modulations, son dénominateur commun peut être formulé ainsi: «Ma vie m'appartient.» Formule qui a son corollaire dans le principe de réciprocité: «La vie des autres leur appartient, je m'interdis d'y porter atteinte.» Cette éthique fait de l'individu son lieu nodal, elle relève donc d'un individualisme mais ce dernier n'a rien en soi d'asocial ou d'égoïste comme le prétendent ses contempteurs. Elle ne décide pas pour et à la place de l'individu, mais en appelle à sa responsabilité et demande à l'Etat de garantir le respect de son choix.

Que penser alors du propos du Dr Kiefer: «Il y a autant de courage et de dignité à affronter ses conditions de vie qu'à se suicider" (cf. l'entretien cité plus haut)? Une fois qu'on se serait assuré que ne s'y cache pas quelque apologie de la "souffrance rédemptrice», alors les tenants d'une éthique de la responsabilité ne peuvent qu'être d'accord: chacun des deux choix a droit à un égal respect. A condition qu'il s'agisse bien, dans chaque cas, d'un choix. Pour le second (le suicide), qu'il exprime la volonté consciente et confirmée de la personne, en dehors de toute pression indue. Pour le premier (affronter ses conditions de fin de vie), qu'il s'agit bien là d'un choix réfléchi - responsable - non l'expression passive d'un laisser-faire!

\section{Références}

1 Vieillir en Suisse. Rapport de la Commission fédérale. Berne; $1995 ; 617-42$

2 A titre d'exemple, cf. Liaudet A, Beauverd M: Une décision éprouvante, in: Rev. Méd. Suisse. 2014;10:1888-9.

3 Citation tirée du corpus de récits de vieillesse réunis dans le cadre des recherches menées au Centre interfacultaire de gérontologie (Université de Genève).

4 Pour le second cas, cf. le quotidien 24 Heures du 23 avril 2014; pour le premier, cf. http://archives.tdg.ch/ex-medecin-cantonal-neuchatel-acquittee-

5 «Le discours d'Exit devient quasi religieux», interview de B. Kiefer, in: Générations-plus.ch, 24 août 2014.

6 Données de l'Office fédéral de la statistique.

7 Lalive d'Epinay C, Cavalli S. Le quatrième âge ou la dernière étape de la vie, PPUF (Le savoir suisse nº3), Lausanne, 2013.

8 Le dossier de cette enquête est consultable sur le site: www.reformiert.info
$\mathrm{CH}-1208$ Genève

christian.lalive[at]unige.ch 